Agnieszka Pater ${ }^{1}$, Aneta Mańkowska-Cyl ${ }^{1}$, Joanna Siódmiak ${ }^{1}$, Agnieszka Jatczak-Gaca², Andrzej Kurylak ${ }^{3}$, Grażyna Sypniewska ${ }^{1}$

${ }^{1}$ Department of Laboratory Medicine, Collegium Medicum, Nicolaus Copernicus University, Bydgoszcz, Poland

2Department of Pediatric Hematology and Oncology, Collegium Medicum, Nicolaus Copernicus University, Bydgoszcz, Poland

${ }^{3}$ Department of Pediatric Nursing, Collegium Medicum, Nicolaus Copernicus University, Bydgoszcz, Poland

\title{
Biomarkers of bone cell activity in children and adolescents with newly diagnosed, untreated acute Iymphoblastic leukemia
}

\author{
Corresponding author: \\ Agnieszka Pater, PhD \\ Department of Laboratory Medicine, \\ Collegium Medicum, Nicolaus \\ Copernicus University, \\ 9 Sklodowskiej-Curie St. \\ 85-094 Bydgoszcz, Poland \\ Phone: 0480525854046 \\ Fax: 0480525853603 \\ e-mail: agnieszka.chrapkowska@wp.pl
}

\begin{abstract}
Introduction. Controversial data on disturbances in mineral homeostasis and bone mass were reported in children with diagnosed untreated acute lymphoblastic leukemia (ALL). Early detection of bone metabolism abnormalities is important for monitoring the effect of therapy on the skeleton. The purpose of this study was to evaluate bone metabolism in children and adolescents with newly diagnosed acute lymphoblastic leukemia by assessing biomarkers of bone cell activity.

Materials and methods. Propeptide of type 1 procollagen (P1NP) and osteocalcin (OC) as bone formation markers and C-terminal telopeptide of type 1 collagen (CTX) and tartrate resistant acid phosphatase $5 \mathrm{~b}$ (TRAP $5 b$ ) as resorption markers were determined in 22 Caucasian children and adolescents (12 boys 4-21 years, 10 girls 4-16 years) with newly diagnosed, untreated ALL and in 22 age- and gender-matched controls.

Results. Bone formation, in particular, and bone resorption were significantly reduced in ALL children and adolescents compared with controls (Me P1NP 51.9 vs. $433.4 \mu \mathrm{g} / \mathrm{L}$ and OC $16.1 \mathrm{vs.} 80.5 \mu \mathrm{g} / \mathrm{L} ; \mathrm{p}<0.0001$; Me CTX 0.454 vs. $1.225 \mu \mathrm{g} / \mathrm{L}$ and TRAP $5 \mathrm{~b} 2.8 \mathrm{vs.} 5.6 \mathrm{U} / \mathrm{L} ; \mathrm{p}<0.001)$. P1NP positively correlated with OC $(r=0.56 ; p=0.01)$ and CTX correlated with TRAP $5 b(r=0.54 ; p=0.02)$ in children and adolescents with ALL. Median P1NP and OC concentrations in ALL children (4-9 years) were dramatically reduced compared with the healthy ones (10-fold and 9-fold respectively), whereas in adolescents with ALL (10-21 years) both bone formation markers were reduced in a lesser degree in comparison with the healthy adolescents. Conclusions. Acute lymphoblastic leukemia influences bone metabolism which is strongly related to the age of onset. More significant disturbances in bone turnover, particularly in bone formation (suppression of collagen synthesis), are observed in children with untreated ALL in comparison with adolescents with ALL. Key words: Acute lymphoblastic leukemia (ALL), bone turnover markers, bone formation, bone resorption
\end{abstract}

Med Res J 2016; 1 (1): 43-47

\section{Introduction}

Acute lymphoblastic leukemia (ALL) is the most prevalent form of curable malignant disease found in children and has increasing numbers of survivors. Disturbances in mineral homeostasis and bone mass in children with diagnosed ALL were reported by Atkinson et al. over 20 years ago [1]. In children with ALL at diagnosis, the predominant features of bone metabolism are: reduced bone mineral density accompanied by suppressed bone formation, reflected by low turnover of bone collagen and other bone formation markers [2]. On the other hand, the data on bone resorption markers are inconsistent showing reduced, normal or increased values [2-6].

Vertebral fractures at diagnosis of ALL in children are an uncommon problem, whereas osteopenia and osteoporosis, as late consequences of treatment in childhood and adolescence, are common. However, the information on the mechanisms involved in abnormal 
bone metabolism is still insufficient. Disturbances in systemic growth hormone axis, hyperleptinemia and vitamin $\mathrm{D}$ deficiency have been reported to play a role in bone remodeling in association with acute lymphoblastic leukemia. Children with ALL show decreased bone volume and diminished number of bone cells [4].

The purpose of this study was to evaluate bone metabolism in children and adolescents with newly diagnosed acute lymphoblastic leukemia by assessing biomarkers of bone cells activity.

\section{Patients and control subjects}

22 Caucasian children and adolescents (12 boys aged 4-21 years, 10 girls aged 4-16 years) with newly diagnosed, untreated acute lymphoblastic leukemia (ALL) recruited from Department of Pediatrics, Hematology and Oncology, Collegium Medicum, Nicolaus Copernicus University were included in the study. We excluded those who suffered from chronic diseases and had taken medicines known to affect bone metabolism. The patients were divided into two groups: ALL children (age range 4-9 years; 8 girls and 4 boys) and ALL adolescents (age range 10-21 years; 2 girls and 8 boys). All patients after diagnosis were treated according to the Non-B ALL BFM 90 protocol.

Age- and gender-matched control group included 12 boys aged $4-21$ years and 10 girls aged 3-16 years (C). Control group consisted of healthy children and adolescents which were recruited from the general population. The inclusion criteria were: height, weight within the $3^{\text {rd }}$ and $97^{\text {th }}$ percentile, lack of systemic illnesses, endocrine or renal disorders, chronic diseases, obesity, no medicines known to affect bone metabolism. None of them was receiving any medication. They showed normal physical development. Controls were also divided into two groups: healthy children (median age 5.3 years; 8 girls and 4 boys) and healthy adolescents (median age 14.4 years; 2 girls and 8 boys).

The study was approved by the Ethics Committee, Collegium Medicum, N.C. University.

\section{Materials and methods}

Venous fasting blood was collected between 7.00 and 9.00 a.m. to avoid any bias from diurnal variation. Blood was centrifuged, immediately after clotting, at $1500 \mathrm{~g}, 4^{\circ} \mathrm{C}$ for $15 \mathrm{~min}$. to obtain serum. Serum samples were frozen at $-70^{\circ} \mathrm{C}$ and stored until the assays were run.

Serum propeptide of type 1 procollagen (P1NP), osteocalcin (OC) as bone formation markers and C-terminal telopeptide of type 1 collagen (CTX) as resorption markers were assayed by ECLIA on clinical chemistry analyzer (Elecsys, Roche Diagnostics). Tartrate resistant acid phosphatase $5 b$ (TRAP 5b) was measured by ELISA (Metra ${ }^{\circledR}$ TRAP5b EIA Kit Quidel Corp.).

Elecsys Total P1NP bone formation marker (total procollagen type $1 \mathrm{~N}$-terminal propeptide) detects the serum concentration of total P1NP and reflects changes in synthesis of new type 1 collagen; detection limit is $5 \mu \mathrm{g} / \mathrm{L}$; measuring range $5-1200 \mu \mathrm{g} / \mathrm{L}$. The OC assay detects total osteocalcin (intact and N-MID fragment) released by osteoblasts to the circulation; detection limit is $0.5 \mu \mathrm{g} / \mathrm{L}$; measuring range $0.5-300.0 \mu \mathrm{g} / \mathrm{L}$. CTX method is based on highly specific monoclonal antibody against a $\beta$-aspartate isomerized form of the sequence EKAHD- $\beta$-GGR derived from the C-terminal telopeptide region of the type I collagen $\alpha-1$ chain; detection limit is $0.01 \mu \mathrm{g} / \mathrm{L}$; measuring range $0.01-6.0 \mu \mathrm{g} / \mathrm{L}$. TRAP $5 \mathrm{~b}$ was determined in serum by immunocapture enzyme assay in which two unique monoclonal antibodies, Trk49 and Trk62, were used. Trk49 is highly specific to inactive TRAP5b fragments. Antibody Trk62 is highly specific to intact, active TRAP5b. Trk49 binds inactive TRAP5b fragments, thereby making Trk62 more available to bind active TRAP5b; assay sensitivity is $0.2 \mathrm{U} / \mathrm{L}$; inter-assay CV 2.0-3.0\% (for activity 3.8-7.4 U/L); intra-assay CV 1.9-2.2\% (for activity 3.4-7.4 U/L).

Data were expressed as medians (25th and 75th percentiles). Normal distribution of the analyzed variables was tested by Shapiro-Wilk test. Spearman rank correlation tests and Mann-Whitney $U$-test were used. $P$ values $\leq 0.05$ were considered statistically significant.

\section{Results}

Bone formation, in particular, and resorption markers were significantly reduced in children and adolescents with untreated acute lymphoblastic leukemia (ALL) compared with healthy children and adolescents. Biochemical bone turnover markers concentrations or activity in study participants were shown in Table 1. The median age was 8.0 (4.0-14.0) yrs for patients with diagnosed ALL and 8.0 (5.0-14.4) yrs for age-matched controls. Patients with acute lymphoblastic leukemia at diagnosis (ALL) had over 8-fold lower P1NP concentration than controls (C). OC concentration at onset of ALL was also very low compared with that of controls (16.1 vs. $80.5 \mu \mathrm{g} / \mathrm{L}, \mathrm{p}<0.0001$ ). Similarly, CTX and TRAP 5b were significantly lower (0.454 vs. $1.225 \mu \mathrm{g} / \mathrm{L}$, $\mathrm{p}<0.001 ; 2.8$ vs. $5.6 \mathrm{U} / \mathrm{L}, \mathrm{p}<0.001$ respectively). P1NP positively correlated with $\mathrm{OC}(r=0.56, \mathrm{p}=0.008)$ and CTX with TRAP $5 b(r=0.54, p=0.02)$ in children and adolescents with ALL. Significant positive correlations were also found between P1NP and OC, CTX, TRAP 5b in healthy children and adolescents $(r=0.67, p=0.002$; 
Table 1. Biochemical bone turnover markers in study participants. Results were expressed as median (25th-75th percentiles)

\begin{tabular}{llll}
\hline & $\begin{array}{l}\text { ALL } \\
\mathbf{n}=\mathbf{2 2}\end{array}$ & $\begin{array}{l}\mathbf{C} \\
\mathbf{n}=\mathbf{2 2}\end{array}$ & $\mathbf{p}$ \\
\hline Gender $(\mathrm{F} / \mathrm{M})$ & $10 / 12$ & $10 / 12$ & $\mathrm{~ns}$ \\
Age $(\mathrm{yrs})$ & $8.0(4.0-14.0)$ & $8.0(5.0-14.4)$ & $\mathrm{ns}$ \\
P1NP $[\mu \mathrm{g} / \mathrm{L}]$ & $51.9(41.5-152.1)$ & $433.4(372.8-748.4)$ & $<0.0001$ \\
OC $[\mu \mathrm{g} / \mathrm{L}]$ & $16.1(7.7-34.1)$ & $80.5(46.7-126.8)$ & $<0.0001$ \\
CTX $[\mu \mathrm{g} / \mathrm{L}]$ & $0.454(0.302-0.607)$ & $1.125(0.952-1.550)$ & $<0.001$ \\
TRAP 5b [U/L] & $2.8(0.7-4.4)$ & $5.6(5.1-7.9)$ & $<0.001$
\end{tabular}

ALL — acute lymphoblastic leukemia; P1NP — propeptide of type 1 procollagen; OC — osteocalcin; CTX — C-terminal telopeptide of type 1 collagen; TRAP $5 b$ - tartrate resistant acid phosphatase $5 b$

Table 2. Biochemical bone turnover markers in children with untreated acute lymphoblastic leukemia (ALL) and healthy controls (C). Results were expressed as median (25th-75th percentiles)

\begin{tabular}{llll}
\hline & \multicolumn{3}{c}{ Children } \\
\cline { 2 - 4 } & $\mathbf{A L L}=\mathbf{1 2}$ & $\mathbf{C}$ & $\mathbf{p}$ \\
\hline Gender $(\mathrm{F} / \mathrm{M})$ & $8 / 4$ & $\mathbf{N}=\mathbf{1 2}$ & $\mathrm{ns}$ \\
Age $(\mathrm{yrs})$ & $4.5(4.0-5.0)$ & $5.3(4.0-6.0)$ & $\mathrm{ns}$ \\
P1NP $[\mu \mathrm{g} / \mathrm{L}]$ & $46.4(41.5-152.1)$ & $435.7(407.6-517.9)$ & $<0,0001$ \\
OC $[\mu \mathrm{g} / \mathrm{L}]$ & $8.5(5.9-23.6)$ & $74.9(65.7-104.0)$ & $<0,0001$ \\
CTX $[\mu \mathrm{g} / \mathrm{L}]$ & $0.422(0.333-0.474)$ & $1.126(0.993-1.550)$ & $<0,0001$ \\
TRAP 5b [U/L] & $3.4(1.3-5.0)$ & $6.4(5.5-7.5)$ & 0,003
\end{tabular}

ALL — acute lymphoblastic leukemia; P1NP — propeptide of type 1 procollagen; OC — osteocalcin; CTX - C-terminal telopeptide of type 1 collagen; TRAP $5 \mathrm{~b}$ - tartrate resistant acid phosphatase $5 \mathrm{~b}$

$r=0.69, p=0.0001 ; r=0.60, p=0.006$ respectively) and between OC and CTX, TRAP $5 b(r=0.76$, $p=0.001 ; r=0.58, p=0,005$ respectively). CTX positively correlated with TRAP $5 b$ in controls $(r=0.53$, $\mathrm{p}=0.02)$.

Next, ALL patients and controls were divided into groups according to age (Tab. 2 and Tab. 3). All determined biochemical bone turnover markers were significantly reduced in prepubertal ALL children compared with controls. Me P1NP was 9.4-fold lower in ALL children compared with the healthy ones $(p<0.0001)$. Similarly, OC concentration was 8.8-fold lower in leukemic children in comparison with healthy children at the same age $(p<0.0001)$. However, in prepubertal group CTX concentration and TRAP $5 b$ activity were only 3-fold lower in ALL children than in controls ( $p<0.0001$ and $p<0.003$ respectively).

Similarly, in adolescents with ALL, P1NP and OC concentrations were almost 6 -fold and almost 5-fold lower ( $p<0.03$ and $p<0.01$ respectively) in comparison with the healthy ones. In ALL adolescents, CTX concentration and TRAP $5 \mathrm{~b}$ activity were significantly lower than in controls $(p<0.03)$.

\section{Discussion}

Bone turnover in children reflects bone remodeling in which bone formation dominates over bone resorption. The pathogenesis of bone loss relates to an imbalance between resorption and formation with the excess of the former over formation of bone [7]. Early detection of abnormalities in bone turnover may be facilitated by assessing biomarkers of bone metabolism, including vitamin D status. Bone turnover markers reflect the actual status of bone metabolism. In many children with cancer, biomarkers of bone formation (osteocalcin, bone specific alkaline phosphatase and carboxy-(or $\mathrm{N}$-terminal) propeptide of type 1 procollagen) were observed to be suppressed, while bone resorption markers were elevated as measured by serum cross-linked (or C-terminal) telopeptide of type 1 collagen. Insulin-like growth factor 1 , which stimulates bone formation, may be suppressed indirectly indicating a growth hormone insufficiency. Leptin may also play a role in bone remodeling as hyperleptinemia has been observed in association with acute lymphoblastic leukemia. Evaluation of bone status using such biomarkers is complicated by 
Table 3. Biochemical bone turnover markers in adolescents with untreated acute lymphoblastic leukemia (ALL) and healthy controls (C). Results were expressed as median (25th-75th percentiles)

\begin{tabular}{llll}
\hline & \multicolumn{1}{c}{ Adolescents } \\
\cline { 2 - 4 } & $\begin{array}{l}\text { ALL } \\
\mathbf{n}=\mathbf{1 0}\end{array}$ & $\mathbf{C}=\mathbf{n}$ & $\mathbf{p}$ \\
\hline Gender $(\mathrm{F} / \mathrm{M})$ & $2 / 8$ & $2 / 8$ & $\mathrm{~ns}$ \\
Age $(\mathrm{yrs})$ & $14.0(12.0-16.0)$ & $14.4(12.0-16.0)$ & $\mathrm{ns}$ \\
P1NP $[\mu \mathrm{g} / \mathrm{L}]$ & $73.3(51.9-121.3)$ & $431.0(99.3-975.3)$ & $<0.03$ \\
OC $[\mu \mathrm{g} / \mathrm{L}]$ & $21.1(16.1-44.6)$ & $100.1(43.6-126.8)$ & $<0.01$ \\
CTX $[\mu \mathrm{g} / \mathrm{L}]$ & $0.485(0.302-0.938)$ & $1.275(0.557-1.510)$ & $<0.03$ \\
TRAP 5b [U/L] & $1.6(0.7-4.2)$ & $5.5(2.4-7.9)$ & $<0.03$ \\
\hline
\end{tabular}

ALL — acute lymphoblastic leukemia; P1NP — propeptide of type 1 procollagen; OC — osteocalcin; CTX — C-terminal telopeptide of type 1 collagen; TRAP $5 \mathrm{~b}$ - tartrate resistant acid phosphatase $5 \mathrm{~b}$

the lack of universally accepted reference values and the variation of age, gender, or pubertal status. Etiologic factors contributing to the observed skeletal morbidities include disease process, chemotherapy (drugs such as glucocorticoids and methotrexate) and radiotherapy. Other factors common to children with cancer, such as chronic inflammation, dietary changes and physical inactivity, must also be taken into account [6] In accordance with other studies, we found dramatically reduced markers of bone formation expressed P1NP and OC concentrations at ALL diagnosis [5, 8-10]. As P1NP reflects the newly synthesized collagen type 1, it suggests that bone formation in this disease in children and adolescents is strongly negatively affected. Sluis et al. [8]. measured bone alkaline phosphatase and P1NP as bone formation markers and they showed significantly lower concentrations at diagnosis, which increased during follow up of the treatment. In children treated for ALL, lower bone alkaline phosphatase may suggest impaired osteoblast differentiation resulting from a direct effect of chemotherapy on bone [9]. Contrary to our results, they showed normal concentration of bone resorption marker - ICTP [8]. Our results, which are similar to those reported by Crofton et al. [9], indicated reduced bone resorption markers (CTX and TRAP5b) at onset of ALL, however, to a much lesser degree than markers of bone formation. Very recent data by Yang et al. [11] suggest that in acute lymphoblastic leukemia the osteogenic differentiation of bone mesenchymal stem cells, from which osteoblasts originate, may be inhibited, which results in a decreased number of osteoblasts.

The low bone turnover may be caused by the disease itself. It seems, however, that in children and adolescents at diagnosis of ALL the coupling between resorption and formation processes is still maintained to some extent in spite of the fact that bone formation appears to be more affected. Osteoporosis and osteopenia are a significant problem in children with acute leukemia not only at diagnosis but also after chemotherapy $[10,12]$.

We are aware of the limitations of this study, small number of participants and lack of bone mineral density (BMD) and vitamin D status data. However, we evaluated bone turnover with the use of the recommended and sensitive biomarkers and analyzed our data in respect of the age of the patients as this is an important factor to be considered for the proper interpretation of bone marker results.

\section{Conclusions}

Acute lymphoblastic leukemia influences bone metabolism which is related to the age of onset. More significant disturbances in bone turnover, particularly in bone formation (suppression of collagen synthesis), are observed in children with untreated ALL in comparison with ALL adolescents.

\section{References}

1. Atkinson SA, Halton JM, Bradley C, Wu B, Barr RD. Bone and mineral abnormalities in childhood acute lymphoblastic leukemia: influence of disease, drugs and nutrition. Int J Cancer 1998; 11 (suppl.): 35-39.

2. Sorva R, Kivivuori SM, Turpeinen M et al. Very low rate of type I collagen synthesis and degradation in newly diagnosed children with acute lymphoblastic leukemia. Bone. 1997; 20: 139-143.

3. Muszyńska-Rosłan K, Konstantynowicz J, Krawczuk-Rybak M et al. Bone mineral density and markers of bone turnover in patients treated for malignant disease in childhood. Med Wieku Rozwoj 2004; 8 : 1041-1054.

4. Leeuw JA, Koudstaal J, Wiersema-Buist J, Kamps WA, Timens W. Bone histomorphometry in children with newly diagnosed acute lymphoblastic leukemia. Pediatr Res 2003: 54: 814-818.

5. Arikoski P, Komulainen J, Riikonen P, Voutilainen R, Knip M, Kröger H. Alterations in bone turnover and impaired development of bone mineral density in newly diagnosed children with cancer: a 1-year prospective study. J Clin Endocrinol Metab 1999; 84: 3174-3181.

6. Atkinson SA. Vitamin D status and bone biomarkers in childhood cancer. Pediatr Blood Cancer 2008; 50 (suppl. 2): 479-482. 
7. Lethaby C, Wiernikowski J, Sala A, Naronha M, Webber C, Barr RD. Bisphosphonate therapy for reduced bone mineral density during treatment of acute lymphoblastic leukemia in childhood and adolescence: a report of preliminary experience. J Pediatr Hematol Oncol 2007; 29: 613-616.

8. van der Sluis IM, van den Heuvel-Eibrink MM, Hählen K, Krenning EP, de Muinck Keizer-Schrama SM. Altered bone mineral density and body composition, and increased fracture risk in childhood acute lymphoblastic leukemia. J Pediatr 2002; 141 204-210.

9. Crofton PM, Ahmed SF, Wade JC, Elmlinger MW, Ranke MB, Kelna $\mathrm{CJ}$, Wallace $\mathrm{WH}$. Bone turnover and growth during and after continuing chemotherapy in children with acute lymphoblastic leukemia. Pediatr Res 2000; 48: 490-496.

10. El-Ziny MA, Al-Tonbary YA, Salama OS, Bakr AA, Al-Marsafawy H, Elsharkawy AA. Low turnover bone disease in Egyptian children with acute leukemia. Hematology. 2005; 10: 327-333.

11. Yang GC, Xu YH, Chen HX, Wang XJ. Acute Lymphoblastic Leukemia Cells Inhibit the Differentiation of Bone Mesenchymal Stem Cells into Osteoblasts In Vitro by Activating Notch Signaling. Stem Cells Int 2015; 2015: 162410. doi: 10.1155/2015/162410.

12. Guren Dolu M, Canbolat Ayhan A, Erguven M, Timur C, Yoruk A, Ozdemir S. Bone mineralization defects after treatment of acute lymphoblastic leukemia ın children. Minerva Pediatr 2015; 67: 419-425. 